\title{
Association study of CCR6 gene single nucleotide polymorphism with susceptibility to rheumatoid arthritis in Iranian population
}

\author{
Yousef Mohammadi Kebar ${ }^{1}$, Massoomeh Akhlaghi ${ }^{1 *}$, Zahra Malekshahi ${ }^{1,2}$, Ahmadreza Jamshhidi ${ }^{1}$, Shayan \\ Mostafaei $^{1}$, Mahdi Mahmoudi ${ }^{1 *}$ \\ ${ }^{1}$ Rheumatology Research Center, Tehran University of Medical Sciences, Tehran, Iran \\ ${ }^{2}$ Department of Immunology, School of Medicine, Tehran University of Medical Sciences, Tehran, Iran
}

\begin{abstract}
Several genome-wide association studies (GWASs) have identified numerous susceptibility genes for risk of rheumatoid arthritis (RA). Moreover, a bulk of the individual association studies in various populations has disclosed that genetics are significantly responsible for RA pathogenesis. CCR6 is a chemokine which is involved in the infiltration of inflammatory cells to sites of immune response. In this study, the association of CCR6 gene rs 1854853 single nucleotide polymorphism (SNP) with susceptibility to RA was evaluated in an Iranian population. The investigated population comprised $250 \mathrm{RA}$ patients and 500 healthy individuals. Real time TaqMan MGB-based PCR allelic discrimination approach was employed to genotype the samples with regard to the CCR6 gene rs1854853 SNP. Considering the A allele of rs1854853 SNP as reference, the $\mathrm{G}$ allele did not demonstrate a different prevalence between RA patients and controls $(p=0.17)$. Moreover, AG and GG genotypes were almost equally distributed between cases and controls ( $p=0.61$ and 0.14 , respectively). Alternately, the dominant model of AG+GG had no significant difference in frequency between the study groups $(p=0.36)$. However, genotypes did show a correlation with the clinicopathological specifications of RA patients. Results suggest that the CCR6 gene rs 1854853 SNP is not involved in the genetic pathogenesis of RA in the Iranian population.
\end{abstract}

Keywords: CCR6 gene, rheumatoid arthritis, single nucleotide polymorphism.

\section{Introduction}

Rheumatoid Arthritis (RA) is an obvious example of a disease encompassing definite features of rheumatologic manifestations with the signs of pain, swelling, and tenderness of joints which are caused by a mixture of autoimmune, genetic, and environmental factors $[1,2]$. RA is characterized by synovium overproliferation, cytokine and chemokine overproduction, autoantibodies such as anti-cyclic citrullinated peptide antibody (Anti-CCP) and rheumatoid factor (RF), angiogenesis, osteoclastogenesis, and cardiovascular and pulmonary complications [3]. In RA, several immune cells such as plasma cells, macrophages, dendritic cells (DCs), and lymphocytes as well as immune complexes infiltrate the synovium environment. Fibroblast-like synoviocytes in RA release various mediators like chemokines, cytokines, and matrix metalloproteinases (MMPs), which degrade the extracellular matrix and eradicate the articular structures in joints $[4,5]$.

To the best of the authors' knowledge and based on numerous studies performed in recent years, autoimmune responses along with genetic materials are the main culprits to be blamed for causing such diseases [6-9]. Genome-wide association studies (GWASs) have clarified new loci or genes that are linked to RA. In this regard, the more new genetic causalities are discovered, the more beneficial new approaches to cure the diseases can be found [10].

As a subset of $\mathrm{CD}^{+} \mathrm{T}$ helper $(\mathrm{Th})$ cells, IL-17producing Th17 cells express CCR6 (CC chemokine receptor 6) on themselves, which is bound to its ligand (CCL20), eventuating in the homing of these cells to inflamed joints. By producing IL-17, a well-known inflammatory cytokine in most autoimmune diseases, Th17 cells play an important role in the pathogenesis of RA [11]. According to studies conducted on RA, some important risk alleles which impact the immune

\footnotetext{
* Corresponding author: Masoomeh Akhlaghi, Email: akhlaghimd@yahoo.com and Mahdi Mahmoudi, Email: mahmoudim@tums.ac.ir, Tele-fax: +98-218-822-0067
}

Received: 21 May 2017; Accepted: 08 August 2017 
system have been revealed [12]. CCR6 gene polymorphisms have been implicated in RA susceptibility [13, 14]. In addition to RA, CCR6 gene SNPs have been associated with Crohn's disease, colorectal cancer, Graves' disease, and psoriasis [1517]. Various SNPs in this gene, including rs3093024, rs3099023, and rs1854853, have been surveyed during recent years, especially in European, Southeast Asian, and African-American populations [18]. A metaanalysis indicated that the CCR6 gene rs 1854853 SNP was associated with an increased risk of RA in different populations [18]. Moreover, a GWAS reported the strong association of rs 1854853 with the risk of RA in Han Chinese and European white populations as well as with the clinical outcomes of the disease [13].

Considering the important role of CCR6 in the immunopathogenesis of RA and previously-observed strong association of CCR6 gene polymorphisms, particularly rs 1854853 , with the risk of RA, the current study evaluated the effect of this SNP on the risk of RA in an Iranian population. Furthermore, the association of genotypes of rs 1854853 SNP with the clinical specifications of RA patients was analyzed.

\section{Materials and Methods \\ Patients and Controls}

From a total of 750 individuals, 250 RA patients (49 males and 201 females) who met 2010 American College of Rheumatology (ACR) classification criteria [19] and 500 healthy controls (113 males and 387 females) were recruited from the Outpatient Rheumatology Clinic of Shariati Hospital. None of the healthy control subjects had autoimmune or rheumatic diseases either in themselves or among their family members. The two groups were matched in terms of age, gender, and ethnicity. The mean age of patients was $50.91 \pm 12.60$ and of controls was $42.49 \pm 12.97$ years. All participants completed informed consent forms with total satisfaction. The study was approved by the Ethics Committee of Tehran University of Medical Sciences. To perform the experiments, $5 \mathrm{ml}$ of peripheral blood was collected from each individual in EDTA-anticoagulated tubes and ESR blood collection test tubes using venipuncture.

\section{Genotyping}

Genomic DNA was extracted from peripheral blood using the phenol-chloroform method [20]. TaqMan MGB-based allelic discrimination technique was employed to genotype the subjects. PCR reaction mixture contained $4.5 \mu \mathrm{l}$ of genomic DNA, $5 \mu \mathrm{l} \mathrm{Taq}$ -
Man Master Mix containing Taq DNA polymerase and dNTPs (Applied Biosystems, Foster City, USA), $0.25 \mu \mathrm{l}$ Taq-Man Genotyping Assay mix containing primers and FAM or VIC labeled probes (Applied Biosystems, Foster City, USA), and distilled water for a final volume of $10 \mu \mathrm{l}$. Thermocyclic conditions of PCR were initially $60{ }^{\circ} \mathrm{C}$ for 30 seconds, then $95{ }^{\circ} \mathrm{C}$ for 10 mins, subsequently 40 cycles of amplification $\left(95^{\circ} \mathrm{C}\right.$ for 15 seconds and $60^{\circ} \mathrm{C}$ for $1 \mathrm{~min}$ ), and finally $60{ }^{\circ} \mathrm{C}$ for 30 seconds.

\section{Statistical analysis}

The chi-square test was utilized to analyze the polymorphism association with the disease. Odds ratio (OR) as the effect size with $95 \%$ confidence intervals $(95 \%$ CI $)$ were measured. Allele frequency and genotype distribution in the control group were evaluated to assess deviations from Hardy-Weinberg equilibrium (HWE). The association of clinical manifestations with the genotypes was also verified applying analysis of variance (ANOVA) using SPSS version 22 (SPSS Inc., Chicago, IL, USA). A $p<0.05$ was considered as statistically significant.

\section{Results}

Table 1 shows the demographic features and laboratory findings of RA patients and healthy control groups. Autoantibodies, including RF and Anti-CCP, were positive in $175(70.1 \%)$ and 160 (64\%) RA patients, respectively. Deformity of the joints was seen in 45 (18\%) patients. Moreover, morning stiffness and arthritis were observed in 120 (48\%) and 140 (56\%) patients, respectively. Measured values for the disease activity score-28 (DAS28), erythrocyte sedimentation rate (ESR), patient global assessment (PGA), visual analog scale (VAS), tender joint counts-28 (TJC28), and swollen joint counts-28 (SJC28) are shown in Table 1.

No significant deviation from the HWE $(p=0.84)$ was observed in the genotype distribution of the CCR6 gene rs 1854853 SNP in the healthy control population (Table 2).

The global major allele of A was assigned as the reference allele for rs1854853 SNP according to the NCBI database (https://www.ncbi.nlm.nih.gov/projects/SNP/). The frequency of the G allele for rs1854853 SNP was less in RA patients than controls $(41.2 \%$ vs. $45.6 \%$, respectively); however, the difference was not significant $(\mathrm{OR}=0.84$, CI: $0.65-1.08 ; p=0.17)$. On the other side, the AA genotype was considered as the reference. The difference in the AG genotype frequencies was not significant between patient and Rheum. Res., Vol. 3, No. 1, Jan. 2018 
control groups $(\mathrm{OR}=0.90, \mathrm{CI}: 0.60-1.35 ; p=0.61)$.

Table 1. Demographic data and laboratory specifications of rheumatoid arthritis patients and healthy controls

\begin{tabular}{lcc}
\hline Characteristic & RA patients $\mathbf{N}=\mathbf{2 5 0}(\boldsymbol{\%})$ & Healthy individuals $\mathbf{N}=\mathbf{5 0 0}(\boldsymbol{\%})$ \\
\hline Male & $49(19.6 \%)^{+}$ & $113(22.6 \%)$ \\
Female & $201(80.4 \%)^{+}$ & $387(77.4 \%)$ \\
Age & $50.91 \pm 12.605^{*}$ & $42.49 \pm 12.971$ \\
Smoker & $3(1.2 \%)^{+}$ & $42(8.4 \%)$ \\
RF (positive) & $175(70.1 \%)^{+}$ & - \\
Anti-CCP (positive) & $160(64 \%)^{+}$ & - \\
DAS28 & $3.33 \pm 1.212^{*}$ & - \\
ESR & $19.87 \pm 17.10^{*}$ & - \\
Deformity (positive) & $45(18 \%)^{+}$ & - \\
Morning stiffness (positive) & $120(48 \%)^{+}$ & - \\
Arthritis (positive) & $140(56 \%)^{+}$ & - \\
PGA & $28.27 \pm 11.97^{*}$ & - \\
VAS & $28.18 \pm 11.87^{*}$ & - \\
TJC28 & $2.89 \pm 3.42^{*}$ & - \\
SJC28 & $2.13 \pm 3.18^{*}$ & - \\
\hline RA, Rh & & - \\
\hline
\end{tabular}

RA, Rheumatoid Arthritis; RF, Rheumatoid Factor; Anti-CCP, Anti-Cyclic Citrullinated Peptide Antibody; DAS28, Disease Activity Score for 28 Joints; ESR, Erythrocyte Sedimentation Rate, PGA, Patient global assessment; VAS, Visual Analogue Scale; TJC, Tender joint count; SJC, Swollen joint count.

* Data are presented as Mean \pm Standard Deviation; ${ }^{+}$Data are presented as $\mathrm{N}(\%)$.

Table 2. Allele and genotype distribution of CCR6 rs1854853 SNP in RA patients and healthy controls

\begin{tabular}{cccccc}
\hline \multirow{2}{*}{ SNP } & \multirow{2}{*}{ Allele /genotype } & RA (N=250) & Control (N=500) & \multirow{2}{*}{ OR (95\% CI) } & \multirow{2}{*}{ value } \\
\hline \multirow{5}{*}{ rs1854853 $(\mathbf{\%})$} & $294(58.8 \%)$ & $544(54.4 \%)$ & Reference & - \\
& A & $206(41.2 \%)$ & $456(45.6 \%)$ & $0.836(0.647-1.081)$ & 0.17 \\
& G & $82(32.8 \%)$ & $143(28.6 \%)$ & Reference & - \\
& AG & $130(52 \%)$ & $256(51.2 \%)$ & $0.90(0.598-1.353)$ & 0.61 \\
HWE & GG & $38(15.2 \%)$ & $101(20.2 \%)$ & $0.66(0.387-1.146)$ & 0.14 \\
\hline
\end{tabular}

RA, Rheumatoid Arthritis; HWE, Hardy-Weinberg Equilibrium

Although the GG genotype was less frequent in RA patients than controls ( $15.2 \%$ vs. $20.2 \%$, respectively), the difference was not statistically significant $(\mathrm{OR}=$ 0.66 , CI: $0.39-1.15 ; p=0.14)$. The AG+GG pattern was the dominant genotype, and it was represented more frequently in controls than in RA patients $(71.4 \%$ vs. $67.2 \%$, respectively); nonetheless, the distribution difference was not significant $(\mathrm{OR}=0.83$, CI: 0.56$1.23 ; p=0.36)$.

The association of clinical manifestations of RA patients according to genotypes of CCR6 gene rs1854853 revealed that the clinical values were not related to variations in genotypes (Table 3 ). The clinical items were RF, Anti-CCP, DAS28, ESR, deformity of joints, morning stiffness, arthritis, PGA, VAS, TJC28, and SJC28, and none of them were associated with rs $1854853 \mathrm{GG}, \mathrm{AG}$, or AA genotypes.

\section{Discussion}

The role of genetics in the onset or development of various types of disease, including autoimmune ones, has been one of the most controversial issues discussed in recently written articles. Researchers all over the world search for probable causal pathogenesis mechanisms in RA. Numerous studies have uncovered new loci in HLA or outside HLA regions that are 
highly related to RA susceptibility, progression, or antirheumatic drugs (DMARDs) [6, 21]. response to drugs such as disease-modifying

Table 3. Association of CCR6 gene rs 1854853 genotypes with various clinical features of the 250 RA patients

\begin{tabular}{lcccc}
\hline \multicolumn{1}{c}{ Characteristic } & GG & AG & AA & $p$ value \\
\hline RF $^{*}$ & $63(36.8 \%)$ & $68(39.1 \%)$ & $53(30.5 \%)$ & 0.613 \\
Anti-CCP* & $19(50 \%)$ & $77(59.2 \%)$ & $64(78 \%)$ & 0.293 \\
DAS28 & $3.43 \pm 1.25$ & $3.22 \pm 1.13$ & $3.45 \pm 1.31$ & 0.409 \\
ESR & $23.62 \pm 24.59$ & $18.33 \pm 14.21$ & $20.56 \pm 17.08$ & 0.282 \\
Deformity $^{*}$ & $4(10.5 \%)$ & $30(23 \%)$ & $12(14.6 \%)$ & 0.146 \\
Morning stiffness $^{*}$ & $23(60.5 \%)$ & $59(45.4 \%)$ & $44(53.6 \%)$ & 0.365 \\
Arthritis & $22(57.9 \%)$ & $66(50.7 \%)$ & $52(63.4 \%)$ & 0.241 \\
PGA & $28.13 \pm 9.98$ & $28.05 \pm 12.19$ & $28.70 \pm 12.22$ & 0.937 \\
VAS & $27.50 \pm 9.837$ & $28.23 \pm 12.07$ & $28.41 \pm 12.55$ & 0.936 \\
TJC28 & $3.41 \pm 3.38$ & $2.60 \pm 2.89$ & $3.10 \pm 4.14$ & 0.412 \\
SJC28 & $1.84 \pm 2.23$ & $2.04 \pm 3.18$ & $2.42 \pm 3.55$ & 0.631 \\
\hline
\end{tabular}

RA, Rheumatoid Arthritis; RF, Rheumatoid Factor; Anti-CCP, Anti-Cyclic Citrullinated Peptide Antibody; DAS28, Disease Activity Score for 28 Joints; ESR, Erythrocyte Sedimentation Rate, PGA, Patient global assessment; VAS, Visual Analogue Scale; TJC, Tender joint count; SJC, Swollen joint count.

Data are presented as number of positive $(\%) ;{ }^{+}$Data are presented as number $(\%)$ in each cell.

RA is depicted as an autoimmune disease with systemic inflammation which specifically manifests as joint destruction and partial involvement of other organs [1]. Most patients experience intense inflammation with exacerbated health conditions over time, showing physical incapacitation and an overrepresentation of the mortality rate compared to the general population [3, 22-24]. Autoantibodies alongside cellular immunity (including $\mathrm{CD}^{+} \mathrm{T}$ cells and related pro-inflammatory cytokines) which are prerequisites for progression of the disease constitute the main players of autoimmune diseases such as RA [3]. Based on recent studies, Th17 cells play a crucial role in RA progression by homing in on inflamed joints and mediating degradation of cartilage. The recruitment of Th17 cells to involved tissues occurs when CCR6 as a cell surface molecule is attached to its ligand, CCL20, which is produced in the synovial cells of RA patients [25]. CCR6 is also expressed on DCs, subsets of B Cells, and $\gamma \delta \mathrm{T}$ cells which produce IL-17, contributing to the inflammation [14].

GWASs and studies of other associations have identified CCR6 gene polymorphisms as a risk factor for RA predisposition. These studies have investigated populations with different ethnic backgrounds, however they represent contradictory results. Although most of them identified a significant relationship between polymorphisms and disease proneness, others did not observe any association [18]. The CCR6 gene rs1854853 association was first discovered in a study on a Han Chinese population [13], as the G allele of this polymorphism was associated with the decreased risk of RA. Moreover, genotypes rs 1854853 depicted a strong association with Anti-CCP-positive, but not with Anti-CCP-negative RA patients [13].

Based on our analysis, the current results are not in accord with some previous studies, and no significant association between CCR6 gene rs1854853 polymorphism and RA risk in the Iranian population was observed. The inconsistency observed in gene association studies can be attributed to several issues. The first and most important one is deemed to be the racial or ethnic disparity between Iranians and other descents, explaining genetic heterogeneity. Another factor that should be considered is the difference in sample sizes among different studies.

In addition to the positive points of this study, such as the fairly large sample size and matching the case and controls with respect to ethnicity to eliminate population stratification bias, there were a number of limitations and caveats. The CCR6 gene has been studied for a number of SNPs in autoimmune diseases. However, this study evaluated only one of them, and that may result in the omission of the concept of interactions between positions which function in an expanded network to cause RA. Moreover, the genetic SNPs of other genes in conjunction with the CCR6 gene as regards immunological functions were not investigated.

Overall, this study investigated the association between the CCR6 gene rs1854853 SNP and a predisposition to RA. No association was found 
between alleles or genotypes of rs1854853 and RA risk. Furthermore, no association was observed between clinical features of RA patients and genotypes of rs1854853 SNP. It seems that the evaluation of this polymorphism in different ethnicities through comprehensive studies like meta-analyses will help researchers achieve a more valid understanding about the role of CCR6.

\section{Acknowledgments}

Those patients who made the accomplishment of this study a reality are kindly acknowledged. This study was underwritten by a financial grant from the Research Deputy of Tehran University of Medical Sciences (Grant No. 96-02-30-325643).

\section{Conflicts of interest}

The author declares no conflicts of interest.

\section{References}

1. Sandoughi M, Kaykhaei MA, Shahrakipoor M, Darvishzadeh R, Nikbakht M, Shahbakhsh S. et al. Clinical manifestations and disease activity score of rheumatoid arthritis in southeast of Iran. Rheumatology Research 2017; 2(2): 61-64. doi: $\quad 10.22631 /$ rr.2017.69997. 1019.

2. Mahmoudi M, Aslani S, Fadaei $R$, Jamshidi AR. New insights to the mechanisms underlying atherosclerosis in rheumatoid arthritis. International Journal of Rheumatic Diseases 2017; 20(3): 289-97. doi: 10.1111/1756-185x.12999.

3. McInnes IB, Schett G. The pathogenesis of rheumatoid arthritis. New England Journal of Medicine 2011; 365(23): 2205-19. doi: 10.1056/nejmra 1004965 .

4. Firestein GS. Evolving concepts of rheumatoid arthritis. Nature 2003; 423(6937): 356-61. doi: 10.1038/nature01661.

5. Bradfield PF, Amft N, VernonWilson E, Exley AE, Parsonage G, Rainger G. et al. Rheumatoid fibroblast-like synoviocytes overexpress the chemokine stromal cell-derived factor 1 (CXCL12), which supports distinct patterns and rates of $\mathrm{CD} 4+$ and $\mathrm{CD} 8+\mathrm{T}$ cell migration within synovial tissue. Arthritis \& Rheumatology 2003; 48(9): 2472-82. doi: 10.1002/art.11219.

6. Kurkó J, Besenyei T, Laki J, Glant TT, Mikecz K, Szekanecz Z. Genetics of rheumatoid arthritis - a comprehensive review. Clinical reviews in allergy \& immunology 2013; 45(2): 170-79. doi: 10.1007/ s12016-012-8346-7.

7. AR J. PADI4 polymorphisms in Iranian patients with rheumatoid arthritis. Acta reumatologica portuguesa 2016; 41(4).

8. Nazari M, Mahmoudi M, Rahmani F, Akhlaghi M, Beigy M, Azarian M. et al. Association of killer cell immunoglobulinlike receptor genes in iranian patients with rheumatoid arthritis. PloS one 2015; 10(12): e0143757. doi: 10.1371/journal. pone. 0143757.

9. Malekshahi Z, Mahmoudi M, Akhlaghi M, Garshasbi M, Jamshidi AR, Nicknam MH. Lack of association between btb domain and cnc homolog 2 polymorphism and susceptibility to rheumatoid arthritis in Iranian population. Indian Journal of Rheumatology 2016; 11(4):197. doi: 10.4103/0973-3698.192682.

10. Prasad P, Kumar A, Gupta R, Juyal RC, Thelma B. Caucasian and Asian specific rheumatoid arthritis risk loci reveal limited replication and apparent allelic heterogeneity in north Indians. PloS one 2012; 7(2): e31584. doi: $\quad 10.1371 /$ journal.pone. 0031584.

11. Hirota $\mathrm{K}$, Yoshitomi $\mathrm{H}$, Hashimoto M, Maeda S, Teradaira S, Sugimoto N. et al. Preferential recruitment of CCR6-expressing Th17 cells to inflamed joints via CCL20 in rheumatoid arthritis and its animal model. The Journal of Experimental Medicine 2007; 204(12): 2803-12. doi: 10.1084/ jem. 20071397.

12. Messemaker TC, Huizinga TW, Kurreeman F. Immunogenetics of rheumatoid arthritis: understanding functional implications. Journal of autoimmunity 2015; 64: 74-81. doi: 10.1016/j.jaut.2015.07.007.

13. Jiang L, Yin J, Ye L, Yang J, Hemani G, Liu AJ. et al. Novel risk loci for rheumatoid arthritis in Han Chinese and congruence with risk variants in Europeans. Arthritis \& rheumatology 2014; 66(5): 1121-32. doi: 10.1002/ art.38353.

14. Kochi Y, Okada Y, Suzuki A, Ikari K, Terao C, Takahashi A. et al. A regulatory variant in CCR6 is associated with rheumatoid arthritis susceptibility. Nature genetics 2010; 42(6): 515-19. doi: 10. 1038/ng.583.

15. Wang K, Zhang H, Kugathasan S, Annese V, Bradfield JP, Russell RK. et al. Diverse genome-wide association studies associate the IL12/IL23 pathway with Crohn Disease. The American Journal of Human Genetics 2009; 84(3): 399-405. doi: 10.1016/j.ajhg.2009.01.026.

16. Rubie C, Kruse B, Frick VO, Kölsch K, Ghadjar P, Wagner M. et al. Chemokine receptor CCR6 expression is regulated by miR-518a-5p in colorectal cancer cells. Journal of translational medicine 2014; 12(1): 48. doi: 10.1186/14795876-12-48.

17. Perkins EA, Landis D, Causey ZL, Edberg Y, Reynolds RJ, Hughes LB. et al. Association of single-nucleotide

polymorphisms in CCR6, TAGAP, and TNFAIP3 with rheumatoid arthritis in African Americans. Arthritis \& Rheumatism 2012; 64(5): 135558 doi: 10.1002/art.33464. 18. Cheng $\mathrm{P}$, Zhang Y, Huang H, 
Zhang W, Yang Q, Guo F. et al. Association between CCR6 and rheumatoid arthritis: a metaanalysis. International Journal of Clinical \& Experimental Medicine 2015; 8(4):5388.

19. Aletaha D, Neogi T, Silman AJ, Funovits J, Felson DT, Bingham CO. et al. Rheumatoid arthritis classification criteria: an American College of Rheumatology/ European League Against Rheumatism collaborative initiative. Arthritis \& Rheumatism 2010; 62(9): 2569-81.

20. Roe BA, Crabtree JS, Khan AS DNA isolation and sequencing. John Wiley \& Sons; 1996.

21. Yamamoto K, Okada Y, Suzuki A, Kochi Y. Genetics of rheumatoid arthritis in Asia [mdash] present and future.
Nature Reviews Rheumatology 2015; 11(6): 375-79. doi: 10.1038/nrrheum.2015.7.

22. Choy E. Understanding the dynamics: pathways involved in the pathogenesis of rheumatoid arthritis. Rheumatology 2012; 51(suppl 5): v3-v11. doi: 10.1093/rheumatology/kes113.

23. Ahmadi H, Jamshidi A, Mahmoudi M, Cuzzocrea S, Fattahi M, Barati A. et al. The potent inhibitory effect of $\beta$-Dmannuronic acid (M2000) as a novel NSAID with immunosuppressive property on anti-cyclic citrullinated peptide antibodies, rheumatoid factor and anti-dsDNA antibodies in patients with rheumatoid arthritis. Current drug discovery technologies 2017; 14(3). doi: $10.2174 / 1570163814666170321$
113059.

24. Mobini M, Mohammadpour RA, Tahmasbi B, Karimi T. Cost comparison of illness in patients with rheumatoid arthritis, osteoarthritis and fibromyalgia syndrome. Rheumatology Research 2017; 2(2): 65-70. doi: $\quad 10.22631 / \mathrm{rr} .2017 .69997$ 1020.

25. Hirota $\mathrm{K}$, Yoshitomi $\mathrm{H}$, Hashimoto M, Maeda S, Teradaira S, Sugimoto N. et al. Preferential recruitment of CCR6-expressing Th17 cells to inflamed joints via CCL20 in rheumatoid arthritis and its animal model. Journal of Experimental Medicine 2007; 204(12): 2803-12. doi: 10.1084/ jem. 20071397 . 